\title{
Anisotropic improved actions
}

\author{
S. Sakai, ${ }^{\mathrm{a}}$, A. Nakamura, ${ }^{\mathrm{b}}$, and T.Saito, ${ }^{\mathrm{c}}$ \\ ${ }^{\text {a}}$ Faculty of Education, Yamagata University \\ ${ }^{\mathrm{b}}$ Research Center for Information Science and Education, Hiroshima University \\ ${ }^{\mathrm{c}}$ Department of Physics, Hiroshima University
}

The studies of the quantum corrections for the anisotropy parameter, $\eta\left(=\xi_{R} / \xi_{B}\right)$, for the improved actions, $\beta\left(C_{0} L\right.$ (Plaq. $)+C_{1} L$ (Rect. $\left.)\right)$, are proceeded in the medium to strong coupling region on anisotropic lattices. The global features for the $\eta$ parameters as a function of $\beta$ and the coefficient $C_{1}$ have been clarified. It has been found by the perturbative analysis that as $C_{1}$ decreases, the slope of the $\eta(\beta)$ becomes less steep and for the actions whose $C_{1}$ is less than $-0.160, \eta$ decreases as $\beta$ decreases, contrary to the case of the standard action. In the medium to strong coupling region, the $\eta$ parameter begins to increase as $\beta$ decreases for all $C_{1}$. This means that for the actions with $C_{1}<-0.160$, the one-loop perturbative results for $\eta$ break down qualitatively and the $\eta$ parameters have a dip. As a result of this dip structure the $\eta$ for Iwasaki's action remains close to unity in the wide range of $\beta$, which means that quantum corrections for the anisotropy parameters are small for it.

\section{INTRODUCTION}

The advantage of using improved actions has been recognized in many area of lattice simulations; at the same time the merits of using anisotropic lattice have been well understood [1]. Then we had started the study of the anisotropic properties for the improved actions 22]. The improved actions we study in this work consist of terms

$$
\left.S_{\mu, \nu}=\beta\left(C_{0} L(\text { Plaq. })_{\mu, \nu}+C_{1} L \text { (Rect. }\right)_{\mu, \nu}\right)
$$

where $L$ (Plaq.) and $L$ (Rect.) represent plaquette and 6-link rectangular loops respectively, and $C_{0}$ and $C_{1}$ satisfies $C_{0}+8 \cdot C_{1}=1$. Our improved action covers

i) tree level Symanzik's action $\left(C_{1}=-1 / 12\right)[3]$,

ii) Iwasaki's action $\left(C_{1}=-0.331\right)$,

iii) QCDTARO's action $\left(C_{1}=-1.409\right)$ [5] etc.

For these classes of improved actions the anisotropic lattice is formulated in the same way as in the case of standard plaquette action[1]. We introduce the coupling constant, $g_{\sigma},\left(g_{\tau}\right)$ and lattice spacing, $a_{\sigma},\left(a_{\tau}\right)$ in space (temperature) direction. With these parameters, the action on the anisotropic lattice is written as,

$$
S=\beta_{\sigma} \cdot S_{i j}+\beta_{\tau} \cdot S_{4 i},
$$

where $\beta_{\sigma}=g_{\sigma}^{-2} \xi_{R}^{-1}, \beta_{\tau}=g_{\tau}^{-2} \xi_{R}$ and $\xi_{R}=a_{\sigma} / a_{\tau}$.

In the weak coupling expansions, the $\eta$ parameter has been written as follows,

$$
\eta=1+N \cdot \alpha\left(\xi_{R}, C_{1}\right) / \beta+O\left(g^{4}\right) .
$$

The behavior of $\alpha\left(\xi_{R}, C_{1}\right)$ had already been reported at lat98[2], which we will show in Fig.1. We have found that $\alpha\left(\xi_{R}, C_{1}\right)$ changes sign around $C_{1} \sim-0.160$. Namely in the weak coupling region, for the Iwasaki's and QCDTARO's actions, the $\eta$ parameter is less than unity contrary to the case of the standard plaquette action.

The natural question is what is the behavior of $\eta$ in the intermediate to weak coupling region, for those improved actions.

\section{NUMERICAL STUDY of $\eta$}

Numerically, the parameter $\eta$ is calculated by the relation $\eta=\xi_{R} / \xi_{B}[6]\left[\begin{array}{l}6] \\ \text {, }\end{array}\right.$, where $\xi_{B}$ is a bare anisotropy parameter which appears in the action,

$$
S=\beta\left(\frac{1}{\xi_{B}} S_{i j}+\xi_{B} S_{i 4}\right),
$$




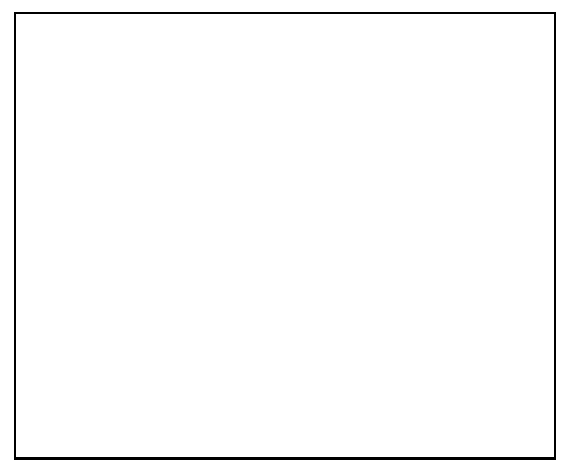

Figure 1. The coefficients $\alpha$ as a function of $C_{1}$

while the renormalized anisotropy parameter is defined by $\xi_{R}=a_{\sigma} / a_{\tau}$. For the probe of the scale for the space and temperature direction, we use the lattice potential in these directions. The lattice potential in temperature direction is defined by Wilson loops in space-temperature plane,

$$
V_{s t}\left(\xi_{B}, p, t\right)=\ln \left(\frac{W_{s t}(p, t)}{W_{s t}(p+1, t)}\right) .
$$

The potential in the space direction is defined in a similar way.

The simulations are mainly done on the $12^{3} \times 24$ lattice for $\xi_{R}=2.0$, and for some $\beta$ on $16^{3} \times 32$ lattice to study the size dependences.

The method of determination of $\eta$ is explained in some detail by the examples of $\beta=4.5$ for Iwasaki's improved action.

We fix $\xi_{R}=2$, and calculate the rate

$$
R\left(\xi_{B}, p, r\right)=\frac{V_{s s}\left(\xi_{B}, p, r\right)}{V_{s t}\left(\xi_{B}, p, \xi_{R} t\right)}
$$

at $\xi_{B}=2.0,2.1,2,2$. The results for $\xi_{B}=2.1$ are shown in the Fig.2 for each $p$ and $r$. For these $R\left(\xi_{B}\right)$ we have made the interpolation by the second order polynomial in $\xi_{B}$ and solve the point where $R=1$ is satisfied. Then the $\eta$ is obtained for each $p$ and $r$. The results for the $\eta$ are shown in Fig.3. It is found that for $p \times r \geq 15$, $\eta$ is almost flat. For the flat region, we take the average of them. The errors are estimated by the
Figure 2. Ratio $R(p, r)$ for $\beta=4.5$ and $\xi_{B}=2.1$

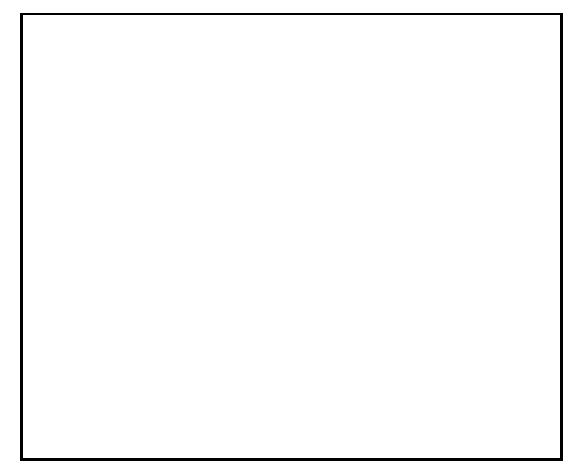

Figure 3. $\eta(p \times r)$ at $\beta=4.5$ and $\xi_{R}=2.0$

Jackknife method. The same analysis has been repeated for other $\beta$ and for the other Improved actions.

In order to check the size dependences, the simulations are carried out on $16^{3} \times 32$ lattice at $\beta=21.0$ and 3.5 for the Iwasaki's improved action. It is found that at $\beta=21.0$ the size dependence is not small but at $\beta=3.5$ it is quite small. Therefore for the intermediate to strong coupling region, the simulation is carried out on $12^{3} \times 24$ lattice. All the results are summarized in Fig.4. 


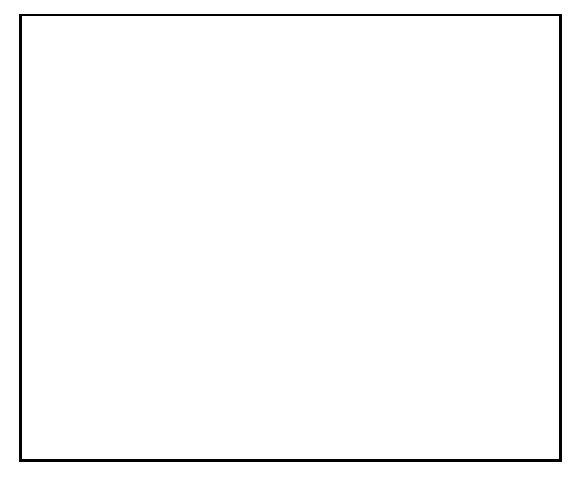

Figure 4. All the results for $\eta\left(\beta, C_{1}\right)$. The results for the standard action is from Ref. 6

\section{CONCLUSIONS AND DISCUSSIONS}

- Iwasaki's Action

The $\eta$ parameter has a shallow dip at $\beta \sim 6.0$ and then blows up towards the small $\beta$ region. The prediction by the one loop perturbative calculation breaks down qualitatively. As a results of this behavior the $\eta \sim 1$ in the wide range of $\beta$.

The results for $\xi_{R}=3$ are also shown in the Fig.4. It is found that the qualitative behavior is the same but the dip around $\beta=6.0$ becomes deeper. This means that the renormalization of the anisotropy parameter is larger for larger $\xi_{R}$.

- Tree-level Symanzik's Action and the action with $C_{1}=-0.16$

The $\eta$ for Symanzik's action is qualitatively the same as those of the standard action[8]; namely it increases monotonically as $\beta$ decreases. But the slope is less steep and then the value of $\eta$ is smaller than the standard action at a given $\beta$.

For the action with $C_{1}=-0.16$, it has been found that $\alpha=0$, namely the renormalization of the anisotropy parameter is zero in the weak coupling perturbative calculations[2]. The $\eta$ in the intermediate to strong coupling region shows similar behavior with the standard plaquette action and Symanzik's action. But again the slope becomes less steep.
- Global features of $\eta\left(\beta, C_{1}\right)$

The calculation with the QCDTARO's action is not carried out yet. But we have found the global structure of the $\eta$ parameter as a function of $\beta$ and $C_{1}$. The interesting point is that the for the action with $C_{1}<-0.16$, the $\eta$ once decreases and then increases with decreasing $\beta$. As a result of this behavior the $\eta$ stays close to one in the wide range of $\beta$ for Iwasaki's improved action. This may be a good features for the numerical simulations.

- Further works

The calculation of the $\eta$ at stronger coupling region and the other $\xi_{R}=0.5,1.5,3.05 .0$ etc are now under study. We are preparing to use the smeared Wilson loops for the simulation in the stronger coupling region.

We are starting the calculation of the lattice spacing $a$ as a function of $\beta$ on the anisotropic lattices.

The behavior of $\eta$ with smaller $C_{1}$ like QCDTARO's action, is also an interesting problem.

We are preparing the simulations of physical quantities on an anisotropic lattice with improved actions. The targets are heavy quark spectroscopy, transport coefficients of quark gluon plasma etc.

\section{ACKNOWLEDGMENTS}

The present work has been done with SX-4 at RCNP and VX-4 at Yamagata. We are grateful for the members of RCNP for warm hospitality and kind supports.

\section{REFERENCES}

1. F.Karsch, Nucl. Phys.B205[FS5](1982), 285

2. S.Sakai, A.Nakamura and T.Saito, Nucl. Phys. B(Proc Suppl)73(1999), 417

3. K. Symanzik, Nucl. Phys. B226(1983), 187

4. Y. Iwasaki, Nucl. Phys. B258(1985), 141; Univ. of Tsukuba preprint UTHEP-118(1983)

5. QCD-TARO Collaboration, Ph. de Forcrand et al. hep-lat/9806008

6. T.R.Klassen hep-lat/9803010

7. G.Burgers et al., Nucl. Phys. B204, 587, 1988 
8. J.Engels, F.Karsch and T. Scheideler heplat/9905002 


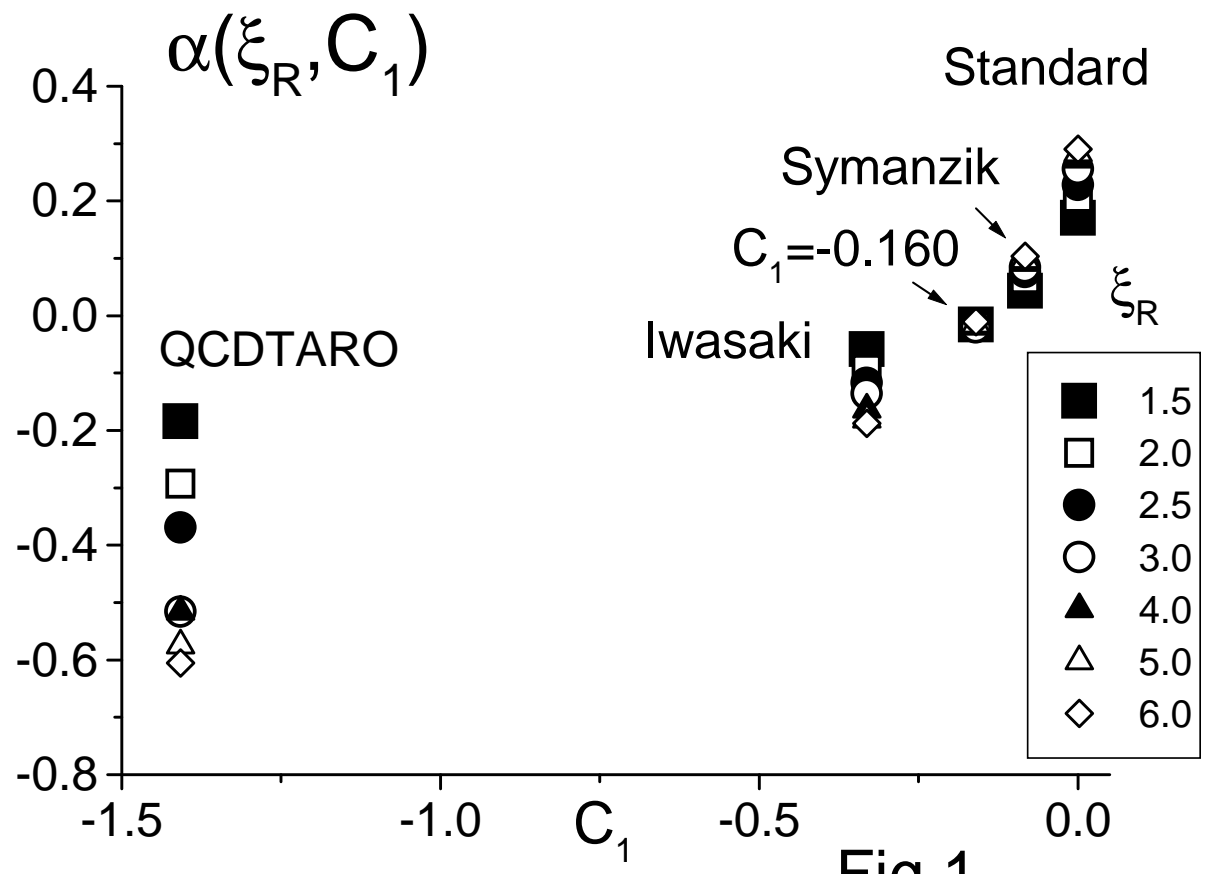

Fig. 1 


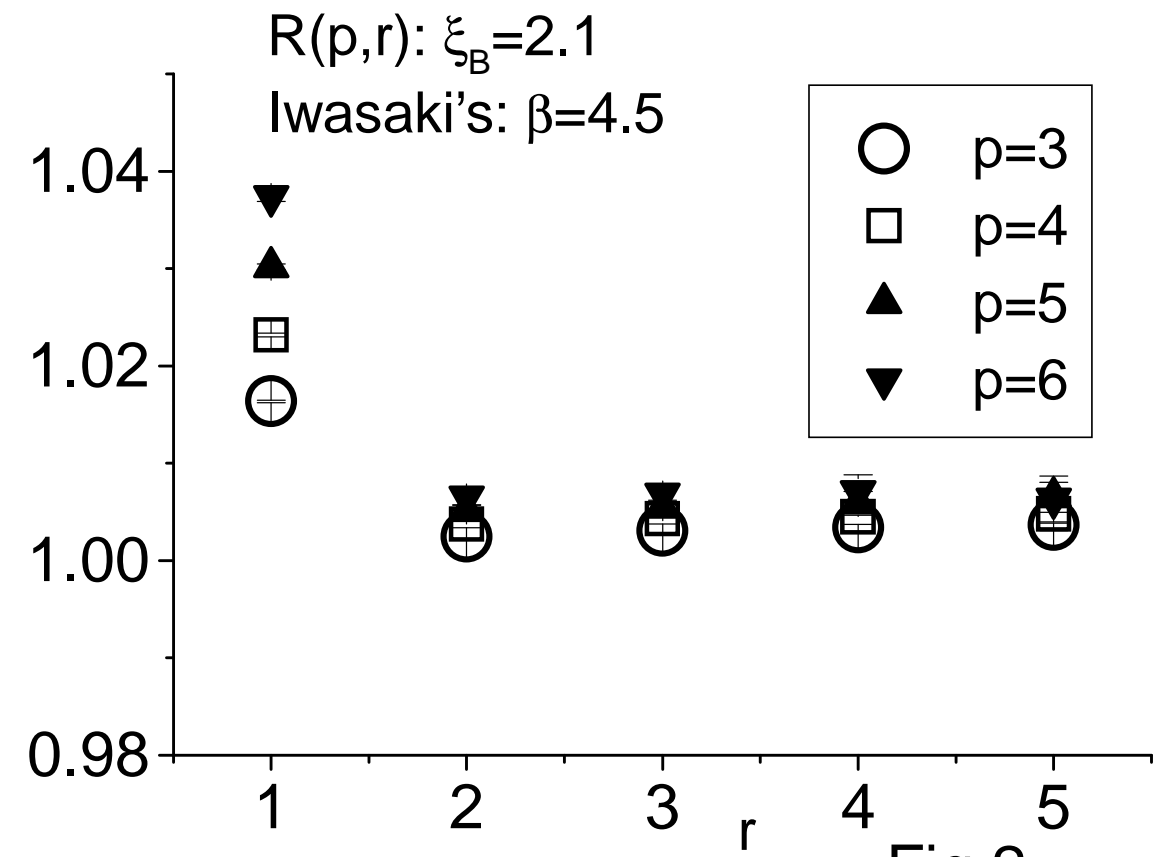

Fig.2 


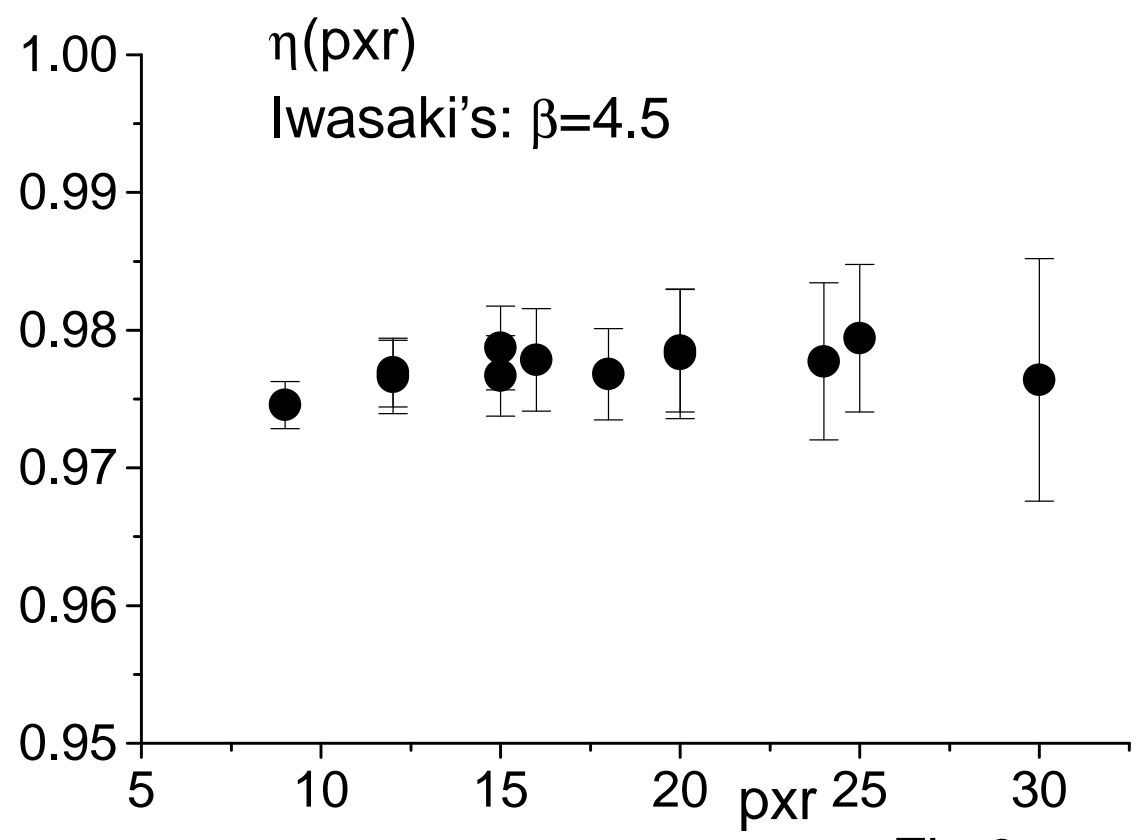

Fig. 3 


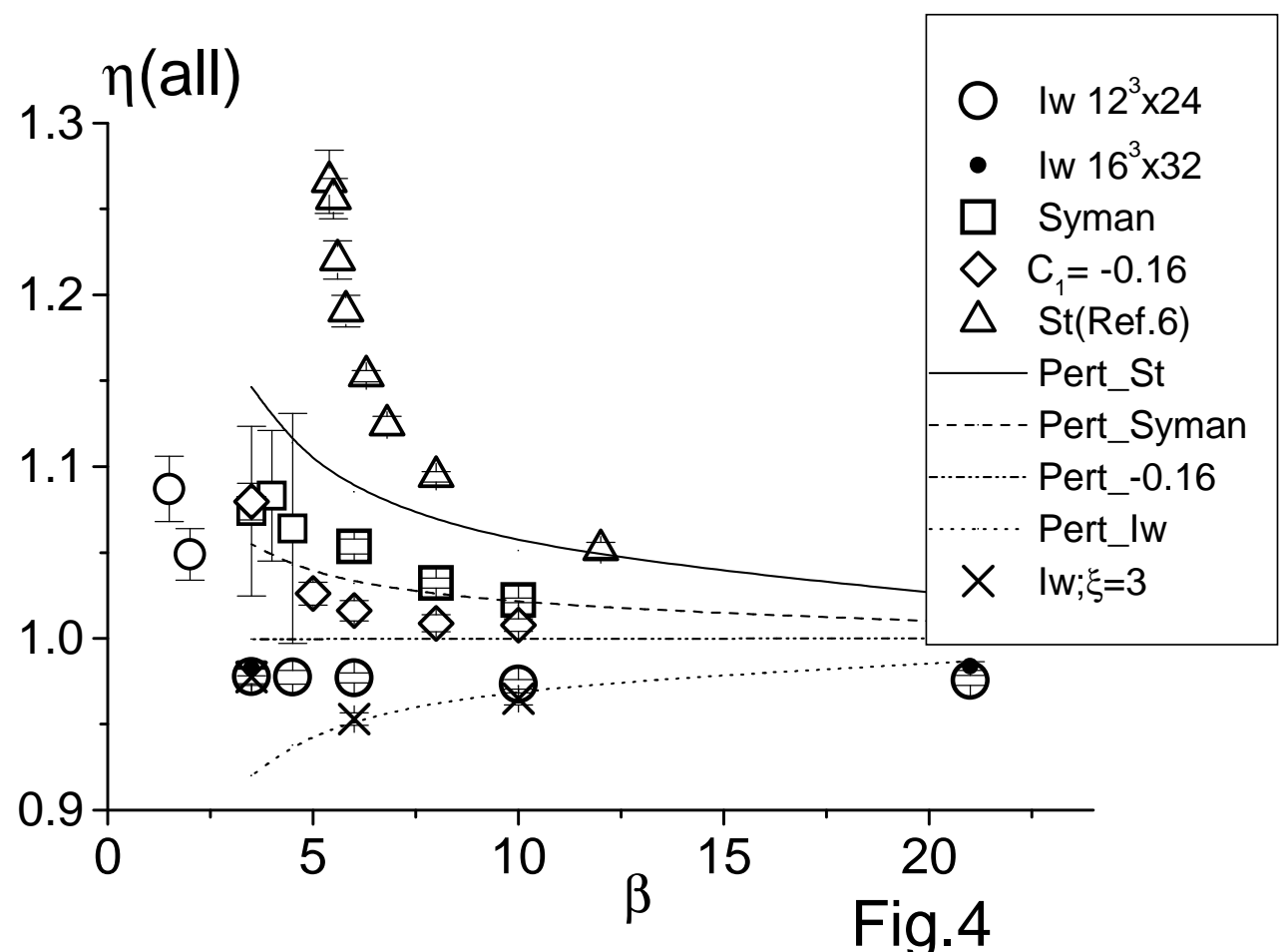

